The author discusses in some detail the possibility that his results might have been due to vitamin $B_{12}$ contained in the liver preparation, and concludes that the evidence points to some other nutritional factor or factors rather than to this nutrient. For example, the liver preparation which was used contained little vitamin $\mathbf{B}_{12}$ and could only have supplied less than I microgram a day compared with the ro or 25 micrograms which have been used by those who have claimed an effect on growth produced by this vitamin. Further, animal experiments showed that vitamin $B_{12}$ added to the stock diet did not produce enhanced growth as did the liver. It seems likely also that the 'growth factor' may easily be lost during preparation of liver, since several other liver powders tested were not active in animal experiments.
Since the publication of this report, the question has been raised as to the advisability of increasing the rate of growth of children by the administration of such preparations. The question implies some confusion as to the causes of increased growth in children. Since liver is a normal article of food, it is reasonable to assume that an effect on growth produced by it indicates that it contains nutrients which were deficient in the diet. In the same way, an improvement in growth following the administration of milk or meat or indeed of a specific nutrient such as vitamin $D$, would imply that the previous diet was also nutritionally deficient. It is therefore difficult to understand what disadvantages might accrue to a child in whom such a deficiency was made good by a dietary supplement.

JoHN YUDKIN

\title{
INFORMATION, MACHINES, AND EXPLANATION
}

\author{
By STEPHen L. SHeRwood
}

\begin{abstract}
'The book stresses the view that man is a product, like so much else, of the play of natural forces acting on the material and under conditions, past and present, obtaining on the surface of our planet.'-Sir Charles Scott Sherrington, Preface to 'Man on his Nature,' I95I, 2nd edition, Cambridge University Press.
\end{abstract}

Man throughout his history has found it necessary to attempt predictions in order to maintain his own life and the continuity of his race. His own needs for survival from day to day, and the forces of nature in his environment, providing or denying opportunities, had to be understood and become capable of being anticipated, so that he could adapt his own behaviour to the greatest number of contingencies and to the greatest advantage.

It is the aim of this paper to outline the means and methods with which such an end could be achieved; this end, stated above, is taken to be axiomatic as an accepted convention: a teleological discussion would lead too deeply into the fields of metaphysics to serve a useful purpose. The data available to us from clinical and psycho- logical observations are often recorded in a language whlch lacks precision because it relies on emotional experience and inference; based on subjective data and analogy it fails to establish an extensive, if not a universal, validity. An attempt is also made to show how the above-named phenomena can be described in terms of the basic sciences and how this enhances accuracy and eases interpretation.

Reaction in response to stimuli is one of the characteristics of living organisms. If we take reaction to mean modification of behaviour, then a stimulus becomes (like a pip on a telegraph wire) a carrier of information or a method by which given conditions and their change are transmitted to the organism. The nature and amount of information one stimulus can supply is variable, depending directly upon the recipient structure and the recipient organism; thus a given ganglion cell can react in one way only: it can transmit an impulse, indicating that something is happening, and that the nature of what is happening represents an adequate stimulus. Thus, a touch corpuscle, when 
stimulated to fire, says ' touched;' if not stimulated, and therefore not firing, ' not touched.' It can be only one of these two predetermined cases, touch being a specific quality. If functioning in conjunction with other touch corpuscles and over a period of time, the information transmitted will comprise complex effects such as intensity, texture (frequency), movement and duration. In other words, if we go one step further and know that the signal coming over a telegraph wire may be not a pip but a dot or a dash, one meaning red the other green, we have, by adding duration as a characteristic to the signal, widened its scope; it can now indicate the presence or absence of either of two qualities. There are other means of transmitting information used both by the nervous system and in telecommunication, such as variation of frequency or amplitude of continuously oscillating structures. The specific information determined by the nature of the receptors constitutes what psychologists call sense data (a sense organ being an apparatus for the reception of stimuli of a specific kind such as light, sound, etc.). Such data, then, are the first pre-requisite stage in the process of understanding. The second is memory or the ability to store sense data. Let it suffice at this point to state that there are several kinds of memory, distinct from each other mainly by their extent in time, and that there is a kind of memory that is transmitted by the organism to its descendants in the form of set typical behaviour patterns in response to given stimuli. Such memory, 'engrams,' are linked to the chromosomes and therefore most likely biochemical. Now, equipped with these two faculties, an animal is still not capable of ' understanding;' the third faculty necessary for that process is the ability to recognize factors common to more than one item of information, that is the ability to generalize or form 'universals,' to define similarities, differences, contrasts and identities. In man this faculty is developed extensively and for a wide range of sense data; it is much less noticeable in other primates and higher mammals; it distinguishes between the capacity to learn and the ability to acquire conditioned reflexes. (Stereotyped simple reactions, depending directly upon the structure of the organism which cannot be modified, are ' built in ' or simple reflexes.)

What, then, is the nature of understanding, and its corollary, explanation? Understanding may be defined as the act of relating sequences of sense data to one another; thus universals or generalizations are formed and these are capable of being applied to new sense data. (In other words, understanding is the act of correlating events.) Explanation, then, is the act of relating new sense data to already ' known' ones, made available by memory, and in doing so provides universals suct as similarities or differences. Deprived of its extent in time the interpretation of facts (as dis tinct from events), with the help of memorye amounts to cognition - an act of lower complexity than understanding, but essential for tha process.

In man's continuous and vital quest for ex⿳亠口冋 planation the devising of models has always bee

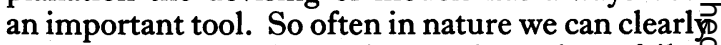
see the starting point and a result, and we fail to see the intervening process, and we hope that if we could but build a gadget which from the same starting point would produce the same result as nature, then we should be able to explain how nature works. (In fact, the legitimacy of this hopo is very much doubted by physicists, who tell us that with a given input and a known output the pos: sibilities of construction of the intervening amplifiee are infinite, so that unless we look into that little black box we do not know how it does it; however, in building something that will do the same as the little black box we may hope to discover some of the essential steps of amplification.) This en deavour is indeed very old and comprises not only the devising of models, but also the comparison of natural processes with known machines. If anatomy we talk readily of pulleys and of levers and we are all familiar with the likeness of the

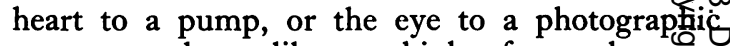
camera, and we like to think of sound wapeos spreading like the waves caused by a pebble in pond, although nobody has ever seen a souni wave. And what modern boy can resist comparing a cuttlefish to a jet engine?

But what kind of machine can do s.omething lik $\overrightarrow{\vec{E}}$ thinking?

Let us return to the three steps which are re quired for understanding. First, reaction to stimuli? as a simple model we may take a switch with ary electric bell. In reacting to the switch being turned. on the electric current makes the bell ring $\frac{\overrightarrow{3}}{3}$ its behaviour, from being silent, has changed into making a sound. In terms of logic and informa tion theory, the bell reacts to a signal. The range of responses is strictly limited-for 'off' and ' on ' we get "no noise ' or ' noise,' or the presences or absence of the sound indicates the position on. the switch. In yet other words, if we know the possibilities are only 'off' or 'on', the answer this mechanism can give is ' yes ' or ' no,' provided the question we ask it is within its possible terms of reference, which are in this case 'Is it on? Similarly, an ordinary desk calculating machine consists principally of a number of axles and $\operatorname{cog} \Phi$ wheels and each time we press a lever on it one of the cogwheels is rotated for a number of cogs corresponding to the order of the depressed lever $\frac{\text { o }}{\mathrm{D}}$ 
each unit step is strictly analogous to our bell model giving an 'on or off' answer. But in the calculating machine we have added our second faculty, memory, because cogwheels on their axles retain their position until the next operation is added. The machine ' remembers' a number of data we feed into it: we code arithmetical propositions with a mechanical device. If such a machine is based on the operations of addition and subtraction in the scale of two we have a Binary Digital Computor. (The Scale of Two is a method of writing numbers using only the numerals 0 and I ; e.g. $2=10,3=1 \mathrm{r}, 4=\mathrm{I} 00$, etc.) Its operations are carried out by stepwise counting processes. Now from this it will appear that each binary digit, or ' bit' as it is nicknamed, represents a unit of information. Let us see what such a machine has in common with a neural mechanism. A single neuron will fire or transmit a signal in obedience to the All-or-None Law in response to an adequate stimulus, i.e. a stimulus above the threshold of excitation of the neuron will transmit one bit. A second neuron upon which our first cell impinges-assuming it has similar characteristics - may either fire and hand on the signal (again providing the arriving stimulus is adequate) or, had it been firing before our impulse arrived, it may stop firing or be inhibited. Let us see further what kind of decisions might be made by three neurons. Let one be called A, another B, and $a$ third, whose action is determined by $A$ and $B, C$. If we accord to $A$ and $B$ the possibilities of exciting or inhibiting $\mathrm{C}$, the following relations may occur :

(I) $\mathrm{C}$ fires if $\mathrm{A}$ or $\mathrm{B}$ or both fire $\quad \ldots \quad\left(\mathrm{X}_{0}\right)$

(2) $\mathrm{C}$ fires if $\mathrm{A}$ but not $\mathrm{B}$ fires .. .. (X)

(3) $C$ fires if B but not A fires .. . . (X)

(4) $C$ fires only if $A$ and $B$ fire.. $\quad \ldots \quad$ (X)

(5) $C$ fires if $A$ or $B$ fire $\quad \ldots \quad$.. (*)

(6) $C$ fires if $A$, whether or not B, fires (X)

(7) $C$ fires if $B$, whether or not A, fires (X:)

(8) $\mathrm{C}$ does not fire if $\mathrm{A}$ and $\mathrm{B}$ do not fire (X)

Each case here represents a unique proposition; one of them, (8), in terms of information transmitted, is trivial. We can symbolize each position as indicated, making use of symbolic logic (a discipline founded by George Boole in the 186 os and developed by Bertrand Russell.) This then permits us to manipulate each proposition as an algebraic term, and we can apply the rules of arithmetic. If we use binary digits the propositions are coded in terms of $I$ and $O$ (it happens or does not happen), and they can be handled by brains or machines.
From the number of possibilities provided by only three neurons the computing that can be done in this way by $10,000,000,000$ cells in the brain would appear to be stupendous, but is it enough to explain all the faculties of the brain? We do not know yet.

A second method of calculation can be usefully invoked to explain certain phenomena, namely, Analogue Computation. Here numbers are represented by magnitudes of physical quantities, such as length, amount of electric current, etc. Calculations are done by combining such quantities according to laws analogous to the laws of the problem to be solved. While calculations in the mode of digital computation are characterized by the operation of counting, as exemplified by an abacus, those of analogue computation are characterized by measuring, as instanced by a slide rule. We know of various factors operating in the organism which are capable of being explained in terms of analogue computation; the metabolic state, the influence of hormones, the emotional state, and the state of nuclear aggregates in the central nervous system which results from ' autorhythmicity'-the tendency to beat of their own accord-and the consequent variability of thresholds and the like are amongst these factors. While the principal advantage of digital computation is accuracy and relative simplicity of design, analogue computation is characterized by greater speed and economy.

A little more needs to be said about memory. A simple desk calculating machine, mentioned before, represents a kind of memory, and so do a gramophone, index cards with punch-holes, and steel tape recorders - there are many other artificial memories used in technology and industry nowadays. In thinking of systems which would explain memory we have.to take account of an apparatus that is capable of learning and, on the other hand, is not destroyed by unconsciousness, ${ }^{\prime}$ whether due to sleep, anaesthesia, trauma or disease. In living organisms several kinds of memory must be presumed: one is the modification of mosaic-like configurations of constituents (such as amino acids) in the protein molecules of the central nervous system; another is enduring 'facilitation' of conduction in associated nervous pathways by the passage of impulses, the former process probably depending upon the latter. Further, we know of neural mechanisms where an impulse travels from its point of origin through a chain of neurons which leads it back to whence it arose, exciting the point of origin afresh and so forth-reverberating or self-exciting circuits. Such circuits can continue as long as metabolism suffices or until a new different stimulus interrupts the passage of the impulses. Now there are two features which are 
highly significant. The reverberating circuit, while it continues, represents a kind of memory, but it is also a kind of abstraction because the unique event defined uniquely in time and space, which set the impulse in motion, becomes generalized once it is no longer fixed as an event in time-it continues as an 'idea' or pattern of excitation fixed only in space, and this idea is now common to all events which are in the form of an original stimulus capable of exciting this reverberating circuit. Here, then, is our third faculty. But this is only one way of forming universals: there are others, such as filters. Just as a sieve will pass only particles of certain size, there are neuronal (and electronic) gadgets which will pass impulses only at a given frequency or strength, and ' size,' 'frequency' and 'strength' are universals. So are the kinds of data received and classified by the very nature of our sense organs--touch, smell, pain, light, and so forth; and within the cerebral cortex there are anatomical arrangements-similar to ' averaging' devices of electronic computing machines--which permit generalizations in the shape of patterns of excitation, so that we can recognize a geometrical shape independent of size or colour, and distinguish a major from a minor chord irrespective of pitch or the instrument upon which it is played; fixed speed of conduction of impulses, reverberating or otherwise, and a constant periodicity of neural oscillations provides us with measurement scales for pitch and speed; and, moreover, these abstractions represent codes which permit us to relate readily new sense data to appropriate items from our memory store. The essential step at which sense data become meaningful is their relation to memory, which must be readily accessible, and this is achieved by classification, coding or the formation of universals, and by correlating universals with one another we obtain rules and laws of a statistical and eventually causal nature with which to make our predictions.

It is well to keep in mind that the sharing of explicit and exact information is largely possible because of our ability to form these universals. Thus, an individual can transform events and facts of practically unlimited complexity into the code of language, using either the written or spoken word, where one can be transformed into the other. Symbolic logic and mathematics are merely specialized codes and techniques of manipulation affording a great amount of precision. In this way the total amount of knowledge theoretically available to any one individual is multiplied by the number of individuals who are able to avail themselves of information. With the enormous strides in the development of telecommunications a new science has been born-Information Theory. It is concerned with the measurement of the amounts of information available and with the efficiency inf communicating information and how it alters, i.e increases, knowledge.

In recent years all the processes indicated herehave been carried out by machines, some as well: as if it had been done by human brains and many more accurately and at an unbelievable speed? There are computing machines of digital an $\frac{\bar{D}}{\mathrm{D}}$. analogue types and the calculations they carros out range from highly complex business and in 2 surance problems to higher mathematics, physic导 and the most complex military problems. There: is a machine which will beat all but the best players at draughts and another at noughts and crosses $\vec{\omega}$ and one that can translate speech into light signals and light signals into speech. This is due to the advances made in physics and electronic engineer? ing, which in turn were possible largely because odo the almost infinite variety of uses to which the thermionic valve can be put. Physiology and ino particular, neurophysiology, owes most of the major recent advances to it.

In the first lines of this paper it was claimed that information and explanation are necessary for op $\overrightarrow{-}$ timal adaptive behaviour, and because the use of the logical machine, as computors have sometimes been called, has been adduced both as an example and as a tool, it is necessary to mention briegly devices whose principle it is to adapt the psrtw formance of a machine to the variations of logid They are called 'governors,' control-, servo-. self-regulating mechanisms. In principle, the measure the output of an engine in relation to 8 set target and vary the power supplied to th engine so that the performance of the engine is adapted to the requirements at any given moment in changing conditions. A thermostat is such device and all modern radio sets have a servo volume control so that the loudness remains even no matter whether the signal received waxes 0 . wanes with atmospheric conditions. Servomechanisms are 'error-actuated '-the greater the deviation from the target, i.e. the greater the error the greater becomes the corrective force. A man attempting to drive a motor car at a constant spee $\widehat{\phi}$ can be considered as a servo-mechanism. The task is indicated by the figure he wishes to attain on the speedometer, and he will supply more fuef by depressing the accelerator pedal or withhold it by taking his foot off in accordance with the difference between actual and desired speedometen reading, which in turn, of course, tends to vary with the inclination of the road. In the field of metabolism and biochemistry, such a system was invoked more than 20 years ago by Walter Cannom in his concept of homeostasis-the ability of the organism to remain adjusted in the chemical and hormonal sense in spite of the continuous dẹ 
mands, stresses and counter-stresses which a living organism encounters. The role of a governor mechanism here was assigned to the autonomic nervous system controlling not only the circulation, but also the endocrines. During the last war K. J. W. Craik was concerned with the complexities of systems whose operation entailed a machine and a human operator. He solved a number of problems by interpreting man's adaptive abilities in terms of servo-mechanisms, by analysing and imitating with machinery accurately the physiological processes involved, in particular sensory adaptation both at the peripheral and central nervous level. By 1945 he had come to the conclusion that servo-mechanisms were, in fact, operative and that the oscillations of the human brain, as seen in the electroencephalogram, were highly suggestive of autorhythmic neural oscillators analogous to the electronic apparatus used in regulating mechanisms. McCulloch, and with him Pitts, began from 1943 onwards to analyse physiological phenomena mathematically; concepts like Gestalt psychology, the clinical syndrome of causalgia and visual following have been satisfactorily explained in terms of basic science. With Wiener's book on 'Cybernetics, or control and communication in the animal and in the machine,' a new method had been established.

What importance has this for the future? $\mathrm{Cy}$ bernetics, in the hands of some workers, has become a superstitious faith rather than a discipline and some of the notions are as unscientific and untenable as they are extravagant, but the new endeavour to explain phenomena of biology and psychology in terms of the most universal of universals, namely mathematics, marks-so the writer believes-a new era. It shares with the atomic age - where, too, mathematical theory came first-potentialities of a golden one. Its expectations for psychology, psychiatry and neurology have already shown justification. To quote McCulloch: '... at the level of theory the most remote of disciplines have developed central ideas that are pulling them together. The wildest speculative analogies of a generation ago turn outz to be similar problems in dissimilar materials $\underset{\mathbb{D}}{\mathbb{D}}$ What is learnt in one field today furnishes profit.- . able attack in the remote tomorrow.' And one other quotation from McCulloch: "The brain is? like a computing machine, but there is no com은 puting machine like the brain.'

None of the ideas and concepts outlined here are claimed as this writer's own. He owes the knowledge to W. S. McCulloch, whose pupil he् had the good fortune to be. Grateful acknowledgment is made to Miss Diana Beck, F.R.C.S. and to the Research Grants Committee of the Middlesex Hospital for the opportunity to acquires this knowledge. Sincere thanks are offered to Donald M. McKay, Reader in Physics at King's. College, for his helpful advice.

It is difficult to provide detailed and specified. references for this paper. The ideas and findingso of several authors overlap and complement each other inextricably. The references given contain $\bar{\omega}$ them in extenso and they are intelligible without expert knowledge in non-medical fields.

\section{BIBLIOGRAPHY}

ADRIAN, E. D. (1947), Brain, 70, 1 .

ADRIAN, E. D. (1949), 'Sensory Integration,' First Sherrington Lecture, University of Liverpool.

BRAZIER, M. A. B. (1948), 'Perspectives of Neuropsychiatry, Lewis, London.

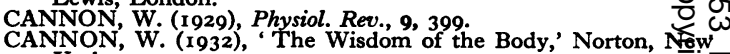

CRAIK, K. J. W. (1943), ' The Nature of Explanation,' Univer Press, Cambridge.

CRAIK, K. J. W. (1947), Brit. F. Psychol., 38, 56.

CRAIK, K. J. W. (1948), Ibid., 38, 142.

LORENTE DE NO, R. (1933), Arch. Neurol. Psychiat., Chicago 30, 245 .

McCULLOCH, W. S. (1945), Bull. Math. Biophys., 7, 89.

MCCULLOCH, W. S. (1946), "The Evolution of the Human Brain,' James Arthur Lecture, Amer. Museum of Natura $\overline{5}$ History, New York.

McCULLOCH, W. S. (1948), Ann. N.Y. Acad. Sci., 50, 259.

MCCULLOCH, W. S., and PFEIFFER, J. (1949), The Scientifio, Monthly, 69, 368.

PFEIFFER, J. (1950), Scientific American, 183, 22. PITTS, W., and McCULLOCH, W. S. (1943), Bull. Math:

RUSSELL, BERTRAND (1948), ‘ An Inquiry into Meaning and Truth,' Allen \& Unwin, London.

WIENER,'N. (r948), 'Cybernetics,' John Wyley, New York.

YOUNG, J. Z. (I950), Reith Lectures, London. Ed. Heinz von Foerster, New York.

\section{RUTHIN CASTLE, NORTH WALES}

A Clinic for the diagnosis and treatment of Internal Diseases (except Mental or Infectious Diseases). The Clinic is provided with a staff of doctors, technicians and nurses.

The surroundings are beautiful. The climate is mild. There is central heating throughout. The annual rainfall is 30.5 inches, that is, less than the average for England.

The Fees are inclusive and vary according to the room occupied.

For particulars apply to THE SECRETARY, Ruthin Castle, North Wales. 